\title{
THE ISO-SWS FLUX STANDARD STARS: SYNTHETIC SPECTRA AND OBSERVATIONS
}

\author{
N.S. VAN DER BLIEK \\ Sterrewacht Leiden, The Netherlands \\ P.W. MORRIS \\ SRON Utrecht, The Netherlands \\ B. VANDENBUSSCHE \\ Instituut voor Sterrenkunde, K.U. Leuven, Belgium \\ L.B.F.M. WATERS AND P. ZAAL \\ Sterrenkundig Instituut Amsterdam, The Netherlands \\ R.A. BELL \\ Astronomy Department, University of Maryland, USA \\ B. GUSTAFSSON AND K. ERIKSSON \\ Uppsala Observatory, Sweden \\ AND \\ TH. DE GRAAUW \\ SRON Groningen, The Netherlands
}

\section{Introduction}

We present flux-calibrated, synthetic spectra for the calibration stars of the Short Wavelength Spectrometer of the Infrared Space Observatory ISOSWS (cf. Kessler et al. 1996). ISO-SWS covers a wavelength range of 2.4 to $45 \mu \mathrm{m}$ (cf. de Graauw et al. 1996), and although in the NIR the flux calibration of Vega can be used, at longer wavelengths the flux calibration has to be extrapolated by other means, because (i) Vega has at $\lambda \geq 25 \mu \mathrm{m}$ an IR excess due to circumstellar dust and (ii) there is an, as yet, unresolved controversy about Vega's flux at $10 \mu \mathrm{m}$ (cf. Rieke et al. 1985). The majority of the standard stars of SWS are of MK class G and K III, as cool giants are amongst the brightest objects in the IR, and model atmospheres for cool giants are available and well-studied (cf. Jørgensen and Gustafsson 1994). 
To obtain flux-calibrated synthetic spectra for these stars we take the following steps: (i) we adopt the absolute calibration of Vega by Mégessier (1995), $F_{555.6 \mathrm{~nm}}=3.56 \times 10^{-23} \mathrm{Wm}^{-2} \mathrm{~Hz}^{-1}$; (ii) we extrapolate this flux calibration into the NIR, with the spectrum of Vega by Dreiling and Bell (1980); (iii) we convert NIR narrowband K photometry of the standard stars into flux densities; and (iv) we extrapolate the flux calibration over the complete wavelength region of SWS by means of synthetic spectra of the standard stars. The $K n$ band is located in a relative clean spectral region of the spectra of $\mathrm{G}$ and $\mathrm{K}$ giants as well as in a clean region of the atmospheric transmission and, moreover, late $\mathrm{G}$ and $\mathrm{K}$ stars emit most of their energy in the NIR.

\section{Synthetic spectra}

Synthetic spectra were generated using the model atmosphere code MARCS (Gustafsson et al. 1975, updated version) and the spectrum generating program by Bell (Bell and Gustafsson 1989; Bell 1993). For Vega we use the model atmosphere by Dreiling and Bell (1980). Stellar effective temperatures $T_{\text {eff }}$ are determined by fitting synthetic colours of a small grid of stellar atmospheres to the observed colours of the stars. Stellar surface gravities and metallicities are taken from the literature. For HR 5755, HR 5981 and HR 7341 no literature data is available, and their parameters are based on their spectral classification.

For each star we have calculated optical and IR colours by folding the synthetic spectra with filter passbands. Zero points were established using the synthetic spectrum of Vega and for the NIR colours the spectra were also folded with the earth's atmospheric transmission. The comparison between observed and synthetic colours is very satisfactory: the average differences are of the same order of magnitude as the observational uncertainties.

We have also calculated the IRAS PSC fluxes and find that the synthetic 12 and $25 \mu \mathrm{m}$ fluxes are systematically too low, although the differences in the $12 \mu \mathrm{m}$ fluxes are well within the observational uncertainties. Bell (1993) calculated IRAS fluxes for G and K giants, whereby he corrected for the geometrical dilution using stellar diameters. His sample includes three of the SWS flux-calibration stars and the comparison with our results is reassuring: the calculated fluxes are, within the observational uncertainties, equal for both studies and the calculated fluxes agree with the observed fluxes.

\section{Uncertainties in the flux-calibrated spectra}

The uncertainties in the continuum fluxes of the calibration stars are of the order 2.5 to $4.5 \%$. This includes: (a) the accuracy of the absolute 
flux calibration of Vega (0.7\% Mégessier 1995); (b) uncertainties in the extrapolation to the NIR. We estimate on the basis of the results of van der Bliek et al. (1996) for their hottest model, that uncertainties in Vega's synthetic spectrum due to uncertainties in the temperature structure of the model atmosphere are of the order of $1.5 \%$; (c) uncertainties in the observed narrowband photometry which are of the order of 0.02 mag.; and (d) the accuracy with which the continuum far-IR fluxes are predicted, which for cool stars is of the order of 2 to $4 \%$ (van der Bliek et al. 1996).

Uncertainties in the detailed spectra, especially for $\lambda \geq 5 \mu \mathrm{m}$, are likely to be larger than the uncertainties quoted here. The discussion by van der Bliek et al. was confined to continuum fluxes; errors in the detailed spectra have not been studied so far. Moreover, IR line lists have, as yet, not been check in detail against high-resolution spectra, and might be found to be far from complete, and need much improvement.

\section{Comparing with the SWS observations}

The spectra of the flux-calibration standard stars are used to derive the photometric responses of the SWS grating detectors in dedicated calibration observations: the measured flux at so-called "key" wavelengths, within a narrow bandpass, is compared with the synthetic spectra (cf. Schaeidt et al. 1996). The instrument calibration and synthetic spectra will be improved iteratively, by comparing the observed and synthetic spectra. In addition, a comparison between observed and synthetic spectra can be used to asses the reliability of the photometric calibration.

Fig. 1 shows such a comparison for $\gamma$ Dra for the CO fundamental ( $\Delta v$ $=1$ ). The observed SWS spectrum was obtained in the slowest AOT1 observing mode, with the full wavelength range and $\lambda / \Delta \lambda \simeq 1500-2000$. The signals from each array of 12 detectors covering the 1st overtone and fundamental bandheads were flatfielded to the median response, sigmaclipped, and rebinned to instrumental resolution. The uncertainty in the relative spectral response is about $2-3 \%$; the uncertainty in the absolute flux levels is of the order of $10-12 \%$. The synthetic spectra presented in Fig. 1 have been rebinned to the instrumental resolution and offseted for clarity.

The overall agreement between the observed and synthetic ${ }^{12} \mathrm{C}^{16} \mathrm{O}$ bandhead profiles is quite excellent. Some mismatch in the weaker ${ }^{13} \mathrm{C}^{16} \mathrm{O} \Delta v=$ 2 bandhead strengths are present, possibly due $T(\tau)$ or $P(\tau)$ of the model atmospheres, or to the ${ }^{12} \mathrm{CO} /{ }^{13} \mathrm{CO}$ ratio. However, this has little affect on SWS calibration over the $0.08 \mu \mathrm{m}$ bandpass, which is centered at $2.48 \mu \mathrm{m}$. 


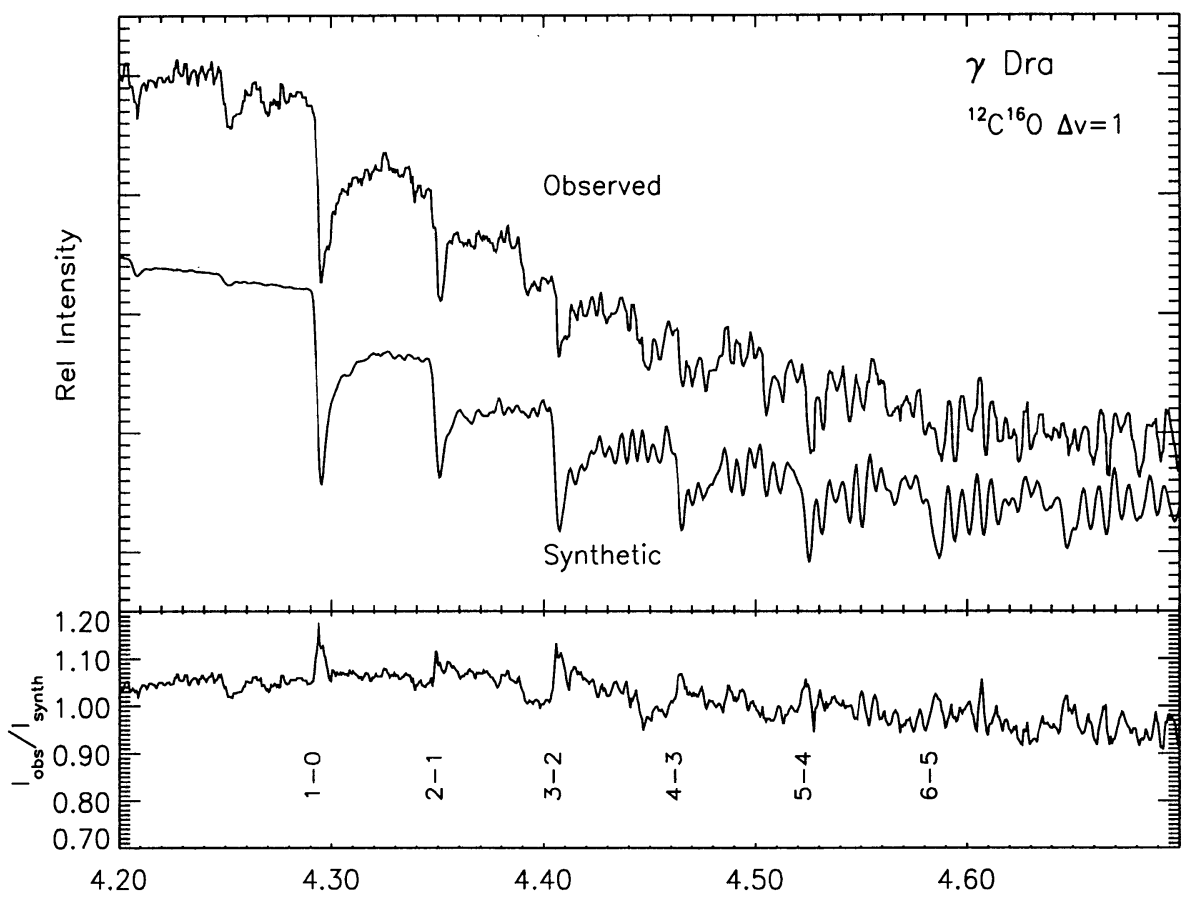

Figure 1. A comparison between observed and synthetic spectra for the fundamental $(\Delta v=1) \mathrm{CO}$ bandheads in the spectrum of $\gamma$ Dra. The top panel shows both the observed (top) and synthetic (bottom) spectrum. The bottom panel shows the observed spectrum relative to the synthetic spectrum, in order to show in more detail the differences between observations and calculations.

Acknowledgements LBFMW acknowledges financial support from the Royal Netherlands Academy of Art \& Sciences KNAW and through a "Pionier" grant from NWO.

\section{References}

Bell R.A. 1993, MNRAS 264, 345

Bell R.A., Gustafsson B. 1989, MNRAS 236, 653

de Graauw T., et al. 1996, A\&A 315, L49

Dreiling L.A., Bell R.A. 1980, ApJ 241, 736

Gustafsson B., Bell R.A., Eriksson K., Nordlund A. 1975, A\&A 42, 407

Kessler M.F., et al. 1996, A\&A 315, L27

Mégessier C. 1995, A\&A 296, 771 (M95)

Rieke G.H., Lebofsky M.J., Low F.J. 1985, AJ 90, 900

Schaeidt S.G., et al. 1996, A\&A 315, L55

van der Bliek N.S., Gustafsson B., Eriksson K. 1996, A\&A 309, 849 